\title{
Novel GFP-like proteins from reef corals with unique light-inducible properties highly suited for fluorescence imaging technologies.
}

\author{
A.Salih* \\ * Electron Microscope Unit, University of Sydney, NSW 2006, Australia.
}

In recent years, there has been a dramatic development of the use of the green fluorescent protein (GFP) homologues, discovered in reef corals and related anthozoans [1], as reporters for the detection of events in living cells and tissues. Numerous GFP-like proteins have been cloned from marine organisms and their expanded use, together with the genetically modified GFPs from the jelly-fish Aequorea, has been paralleled by the development of various photolabeling techniques and of advanced fluorescence-based imaging technologies [3]. Photolabeling includes the use of the photoactivatable PA-GFP [3]; greening properties of DsRed by photobleaching [4]; green to red photoconverting coral protein, Kaede [5]; and kindling of the non-fluorescent anemone protein to red fluorescent state [6]. Research of the diversity, optical properties and biological functions of the GFP-like proteins of the Great Barrier Reef (GBR) corals [7] identified the existence of spectrally diverse blue to red GFP-like proteins in numerous species, many possessing unique photo-inducible properties [8]. Previous research of their localization in coral cells found heterogeneous mixtures of different GFP spectral variants present, functioning in photoprotection by dissipating excessive sunlight via energy transfer cascades [7]. Photoactivation of GFPs in tissue samples of various Great Barrier Reef coral species were tested by exposing frozen or live, small coral samples to epifluorescent light. Acropora millepora, was found to be one of species whose GFP-pigmented tissues exhibited photoactivation. To determine the photoinducible properties of its individual GFP-like proteins, cloned and purified proteins (amilFP513 \& amilFP593 - provided by M. Matz, University of Florida) in phosphate buffer were dried onto glass slides and exposed to various wavelengths (1 min) at epifluorescent or confocal $(458,488,543 \mathrm{~nm})$ irradiation. They were next imaged and spectrally scanned (obj. PL APO 40.0x0.75) by Leica TCS SP2 confocal microscope.

Photoactivation occurred in amilFP513, with green emissions increasing by $\sim 2$ fold following UVA epifluorescent irradiation for $2 \mathrm{~min}$, with no further increase after $4 \mathrm{~min}$ (Fig. 1). Spectral scanning at $488 \mathrm{~nm}$ excitation caused quenching of fluorescence observable as only slight emission increase at 513 $\mathrm{nm}$ after UVA exposure (Fig. 1d). Bleaching by $488 \mathrm{~nm}$ for $1 \mathrm{~min}$ caused a dramatic photoconversion of the red amilFP594, with a reduction of $594 \mathrm{~nm}$ peak and an increase of $535 \mathrm{~nm}$ yellow-green peak (Fig. 2). Thus, UV photoactivation of amilFP513 appears similar to the UV photoactivation of PAGFP, genetically modified for this purpose [4]. Photoconversion of amilFP594 appears to be similar to "greening" of the DsRed coral protein [4], which has emission maxima at 509 (weak) and $583 \mathrm{~nm}$ (strong) [1]. In DsRed, greening is caused by the selective bleaching of the red species of the tetramere by multi-photon wavelengths, causing the fluorescence of the green species, normally quenched by Forster resonance energy transfer, to be enhanced [4]. Photoactivation of PA-GFP and DsRed's "greening" are used as powerful techniques for regional optical marking of cells and proteins and for analyzing protein kinetics within cells $[3,4]$. The discovery of the Great Barrier Reef GFPlike proteins with novel optical properties expands the scope of the available bio-imaging techniques for studying cellular and protein dynamics and in their applications in biotechnology.

References 
[1] M.V. Matz et. al. Nature Biotech. 17, 969-973 (1999).

[2] R. Y. Tsien. Annu. Rev. Biochem. 67, 509-544 (1998).

[3] G. H. Patterson Science, 297, 1873-1877 (2002).

[4] J. S. Marchant et al. Nature Biotech. 19, 645-649 (2001).

[5] R. Ando et al. Proc. Nat. Acad. of Sci. U SA. 99:12651-12656, 2003.

[6] D. M. Chudakov et al. Nature Biotech. 21, 452-452 (2003).

[7] A. Salih et al. Nature, 408, 850-853 (2000).

[8] A. Salih et al. SPIE Proceedings 4963, 194-200 (2003).

[9] Project supported by The University of Sydney SESQUI fellowship to Anya Salih.
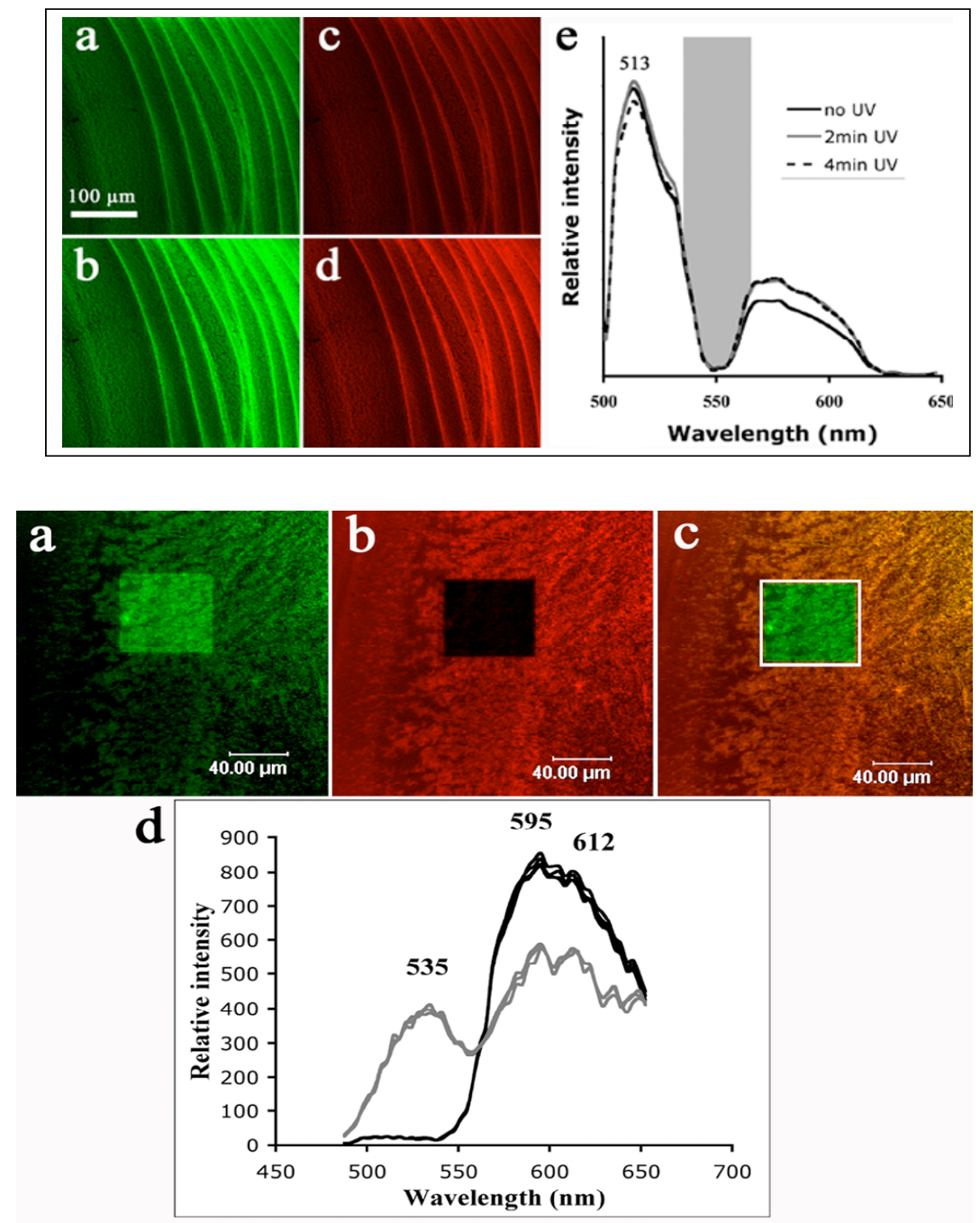

FIG. 1. Photoactivation of green GFP-like coral protein by UVA (360-390 nm). Green emission at $500-520 \mathrm{~nm}$ imaged in PMT1 at excitation by $488 \mathrm{~nm}$ laser line (a, b); red emissions at $570-620 \mathrm{~nm}$ imaged in PMT2 at excitation by $543 \mathrm{~nm}$ laser line (c, d). Emissions before $(a, c)$ and after (b, d) UVA irradiation for 2 min. (d) Emission spectra before and after $2 \& 4 \mathrm{~min}$ UV exposure, spectrally scanned at $488 \mathrm{~nm}$ excitation which appeared to quench UVA-induced fluores-cence amplification (Shaded area indicates excitation 488/543/633 beam splitter position on spectrum)

FIG. 2. Photoconversion of red GFP-like protein amilFP594 in selected AOI to yellow-green color by $488 \mathrm{~nm}$ laser line. Bleaching achieved by zooming to $18 \mu \mathrm{m} \times 18 \mu \mathrm{m}$ area, scanning for 1 min and zooming out to perform imaging and spectral collection. (a) Emissions imaged at 500-540 nm by $488 \mathrm{~nm}$ excitation; and (b) and at $580-650 \mathrm{~nm}$ by $543 \mathrm{~nm}$ excitation, showing increase of yellowgreen and loss of red emissions in AOI; (c) over-layed a and b. (d) Spectral scan at 2-photon $925 \mathrm{~nm}$ excitation shows the appearance of $535 \mathrm{~nm}$ peak in the bleached area (grey line) compared to unbleached area (black line) . 